\title{
Reduced Glutathione Acts to Lessen Radiation Damage in TEM
}

\author{
William H. Massover
}

\section{Department of Biological Sciences, Rutgers University - Newark, 195 University Ave., Newark, NJ. 07102, USA}

Radiation damage to protein specimens during transmission electron microscopy (TEM) and diffraction (TED) remains a big concern in the quest for truly high resolution structure. Low-dose imaging and cryogenic specimen temperatures are common countermeasures [1], but only partially protect against radiation damage. The electron irradiation-induced bubbling response of a thin amorphous layer of dried sodium phosphate buffer provides a very sensitive and directly detectable indicator for radiation damage [2,3]. This experimental study investigates the effect of the major cellular anti-oxidant, reduced glutathione [4], upon irradiation-induced bubbling.

Thin platelets of bovine liver catalase, and a stock solution of sodium phosphate buffer

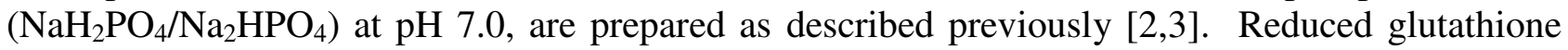
(cat. no. G4251: Sigma-Aldrich, St. Louis, MO. 63187) is dissolved in pure water immediately before use. Aliquots of $2-4 \mathrm{X}$ stock solutions are mixed to give final concentrations of $150 \mathrm{mM}$ buffer, with or without $274 \mathrm{mM}$ glutathione; a small volume of protein-A-coated $15 \mathrm{~nm}$ colloidal gold particles (Ted Pella, Inc., Redding, CA. 92037) is added as a focusing aid. A 16-18 $\mu \mathrm{L}$ droplet of the experimental or control mixture is applied to wet catalase crystals held on a thin formvar support film overlying 400-mesh copper grids; after draining fully, the grids are rapidly dried in air. Grids are examined at room temperature $\left(21^{\circ} \mathrm{C}\right)$ with no cryo-protection. Digitized images are recorded with a 4k x 4k CCD camera (Ultrascan: Gatan, Inc., Pleasanton, CA. 94588) on a Tecnai12 transmission electron microscope (FEI Co., Hillsboro, OR. 97124) operating at 120kV; low-dose or high-dose (10X dosage) exposures are controlled by Tecnai-G ${ }^{2}$ software (FEI).

Images of the dried buffer by itself show numerous irradiation-induced bubbles with low-dose (or high-dose) exposures (Fig. 1a). Addition of $274 \mathrm{mM}$ reduced glutathione to this buffer greatly diminishes the extent of bubbling (low-dose: Fig. 1b; high-dose: Fig. 1c). Sometimes there are no bubbles at all. Even the deeper thickness of dried buffer in the meniscus surrounding catalase crystals often does not show the usual bubble formation with high-dose exposures; very thick regions do form some bubbles with high-dose irradiation, but this is less vigorous than usual. Lower concentrations of reduced glutathione give less of an anti-bubbling effect. Catalase crystals all reveal their characteristic $87 \AA$ major periods (Fig. 2) with sodium phosphate buffer plus reduced glutathione, since this buffer also is a light-atom negative stain [2]; the crystals are clean, wellcontrasted, and show fine details within the ordered protein lattice (Fig. 2).

Observation of a greatly diminished bubbling response in dried sodium phosphate buffer with addition of $274 \mathrm{mM}$ reduced glutathione indicates that the anti-oxidant is effectively countering radiation damage from TEM. Its effect is concentration-dependent. This anti-damage activity should be most valuable for the first low-dose exposure, since additional radiation will soon neutralize all of the anti-oxidant around protein specimens. Addition of reduced glutathione does not interfere 
with imaging protein specimens prepared by unconventional negative staining. It is hoped that reduced glutathione also will be a useful ingredient for future development of multi-component mixtures that will form a much more protective and supportive environment for cryo-TEM than does the usual dilute buffer or pure water. The actual extent whereby reduced glutathione and other [5] radioprotectants are able to decrease radiation damage and prolong the dose tolerance of native protein structure during TEM and TED now must be evaluated quantitatively.

\section{References -}

[1] W. H. Massover, J. Synchrotron Rad. 14 (2007) 116-127.

[2] W. H. Massover, Microsc. Microanal. 14 (2008) 126-137.

[3] W. H. Massover, Microsc. Microanal. 16 (2010) in press.

[4] A. J. Forman, H. Zhang, A. Rinna. Mol. Aspects Med. 30 (2009) 1-12.

[5] W. H. Massover, Proc. $16^{\text {th }}$ Internat. Microsc. Cong. 1 (2006) 493.

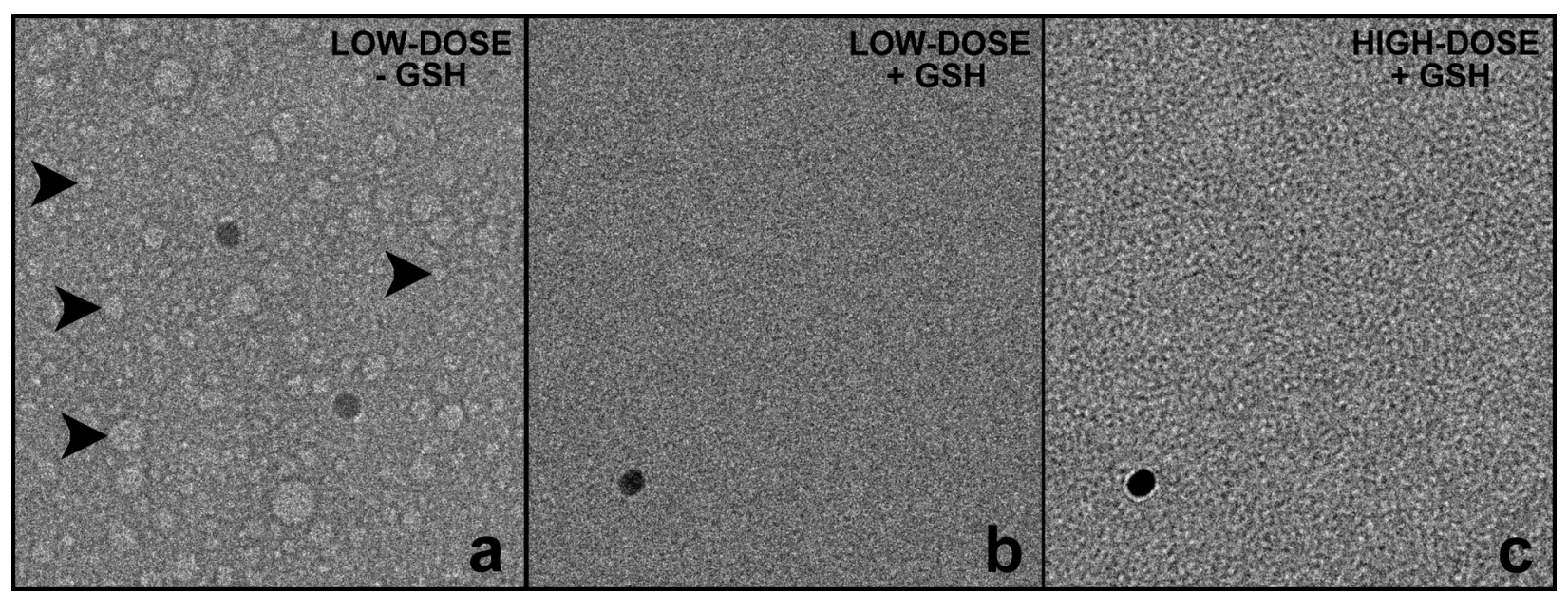

FIG. 1. Low-dose (a,b) and high-dose (c) images with 150mM buffer. (a): Numerous bubbles (e.g., arrowheads) form in pure buffer. (b,c): No bubbles form with added 274mM reduced glutathione (GSH). Thickness and dosage in (a) and (b) are similar. Dark dots are 15nm colloidal gold particles.

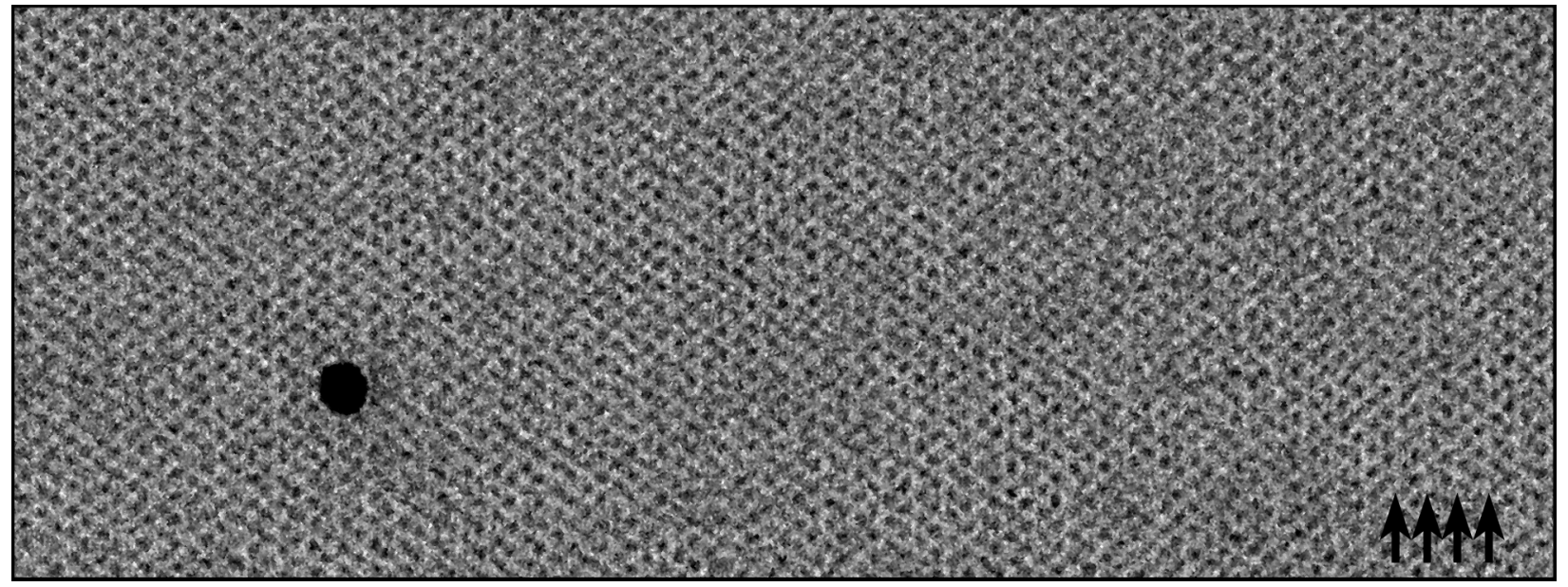

FIG. 2. Lattice image of catalase crystal within buffer containing $274 \mathrm{mM}$ reduced glutathione. Protein is light. Major periods (e.g., arrows) are $87 \AA$ A. Large dark dot is $15 \mathrm{~nm}$ colloidal gold particle. 